\title{
34.
}

\section{NOTE ON THE MAXIMA AND MINIMA OF FUNCTIONS OF THREE VARIABLES.}

[From the Cambridge and Dublin Mathematical Journal, vol. I. (1846), pp. 74, 75.]

If $A, B, C, F, G, H$, be any real quantities, such that

$$
B C+C A+A B-F^{2}-G^{2}-H^{2},
$$

and

$$
(A+B+C)\left(A B C-A F^{2}-B G^{2}-C H^{2}+2 F G H\right)
$$

are positive; the six quantities

$$
B C-F^{2}, C A-G^{2}, A B-H^{2}, A K, B K, C K,
$$

(where $K=A B C-A F^{2}-B G^{2}-C H^{2}+2 F G H$ ) are all of them positive. It is unnecessary to point out the connection of this property with the theory of maxima and minima.

To demonstrate this, writing as usual

$$
\begin{array}{ll}
B C-F^{2}=A^{\prime}, & G H-A F=F^{\prime \prime}, \\
C A-G^{2}=B^{\prime}, & H F-B G=G^{\prime}, \\
A B-H^{2}=C^{\prime}, & F G-C H=H^{\prime},
\end{array}
$$

and $K$ as above: then if $A^{\prime \prime}, B^{\prime \prime}, C^{\prime \prime}, F^{\prime \prime}, G^{\prime \prime}, H^{\prime \prime}, K^{\prime}$ be formed from $A^{\prime}, B^{\prime}, C^{\prime}, F^{\prime}, G^{\prime}, H^{\prime}$, as these and $K$ are from $A, B, C, F, G, H$, we have the well-known formulæ

$$
\begin{array}{ll}
A^{\prime \prime}=K A, & F^{\prime \prime}=K F, \quad K^{\prime}=K^{2} . \\
B^{\prime \prime}=K B, & G^{\prime \prime}=K G, \\
C^{\prime \prime}=K C, & H^{\prime \prime}=K H,
\end{array}
$$


34] NOTE ON THE MAXIMA AND MINIMA OF FUNCTIONS OF THREE VARIABLES. 229

It is required to show that if $A^{\prime}+B^{\prime}+C^{\prime \prime}$ and $A^{\prime \prime}+B^{\prime \prime}+C^{\prime \prime}$ are positive, $A^{\prime}, B^{\prime}, C^{\prime}$, $A^{\prime \prime}, B^{\prime \prime}, C^{\prime \prime}$ are so likewise.

Consider the cubic equation

$$
\left(A^{\prime}-k\right)\left(B^{\prime}-k\right)\left(C^{\prime}-k\right)-\left(A^{\prime}-k\right) F^{\nu_{2}}-\left(B^{\prime}-k\right) G^{\prime 2}-\left(C^{\prime}-k\right) H^{\prime 2}+2 F^{\prime} G^{\prime} H^{\prime}=0,
$$

the roots of which are all real. By the formulæ just given this may be written

$$
k^{3}-k^{2}\left(A^{\prime}+B^{\prime}+C^{\prime}\right)+k\left(A^{\prime \prime}+B^{\prime \prime}+C^{\prime \prime}\right)-K^{2}=0 ;
$$

and the terms of this equation are alternately positive and negative; i.e. the roots are all positive. Hence the roots of the limiting equation

$$
\left(B^{\prime}-k\right)\left(C^{\prime}-k\right)-F^{\prime 2}=0
$$

are positive, i.e. $B^{\prime}+C^{\prime}$ and $B^{\prime} C^{\prime}$ are positive: but from the second condition $B^{\prime}, C^{\prime}$ are of the same sign: consequently they are of the same sign with $B^{\prime}+C^{\prime}$, or positive. Also $A^{\prime \prime}=B^{\prime} C^{\prime}-F^{\prime 2}$ is positive. Similarly, considering the other limiting equations, $A^{\prime}, B^{\prime}, C^{\prime}, A^{\prime \prime}, B^{\prime \prime}, C^{\prime \prime}$ are all of them positive.

In connection with the above I may notice the following theorem. The roots of the equation

$$
\begin{aligned}
(A-k a)(B-k b)(C-. c k)-(A-k a)(F-k f)^{2}-(B-k b)(G-k g)^{2}-(C-k c)(H-k h)^{2} \\
+2(F-k f)(G-k g)(H-k h)=0
\end{aligned}
$$

are all of them real, if either of the functions

$$
\begin{aligned}
& A x^{2}+B y^{2}+C z^{2}+2 F y z+2 G x z+2 H x y, \\
& a x^{2}+b y^{2}+c z^{2}+2 f y z+2 g x z+2 h x y,
\end{aligned}
$$

preserve constantly the same sign. The above form parts of a general system of properties of functions of the second order. 